\title{
Wing Mass Formula for Twin Fuselage Aircraft
}

\author{
Sergei V. Udin* and William J. Anderson $\dagger$ \\ University of Michigan, Ann Arbor, Michigan 48109
}

\begin{abstract}
A formula is derived to calculate structural wing mass. This formula can be applied to twin fuselage aircraft, conventional single-body aircraft and some other unconventional aircraft (such as the Voyager). The approach is particularly useful in the first stages of preliminary aircraft design and in optimization programs where the wing-mass calculation time is an important characteristic. The concept model assumes a nontapered inboard wing section, a tapered outboard wing section and fuel stored only in the outboard wing. The theory for the wing-mass estimation is described. Unlike the other mass formulae where mass spanwise distribution is considered by an "unloading coefficient," the present method integrates the mass spanwise distribution with the air load spanwise distribution. This allows more precise consideration of the wing geometry and mass unloading. There are no simplifications applied and the formula completely reflects the initial concept model. Good comparison with statistical data for single body aircraft is obtained.
\end{abstract}

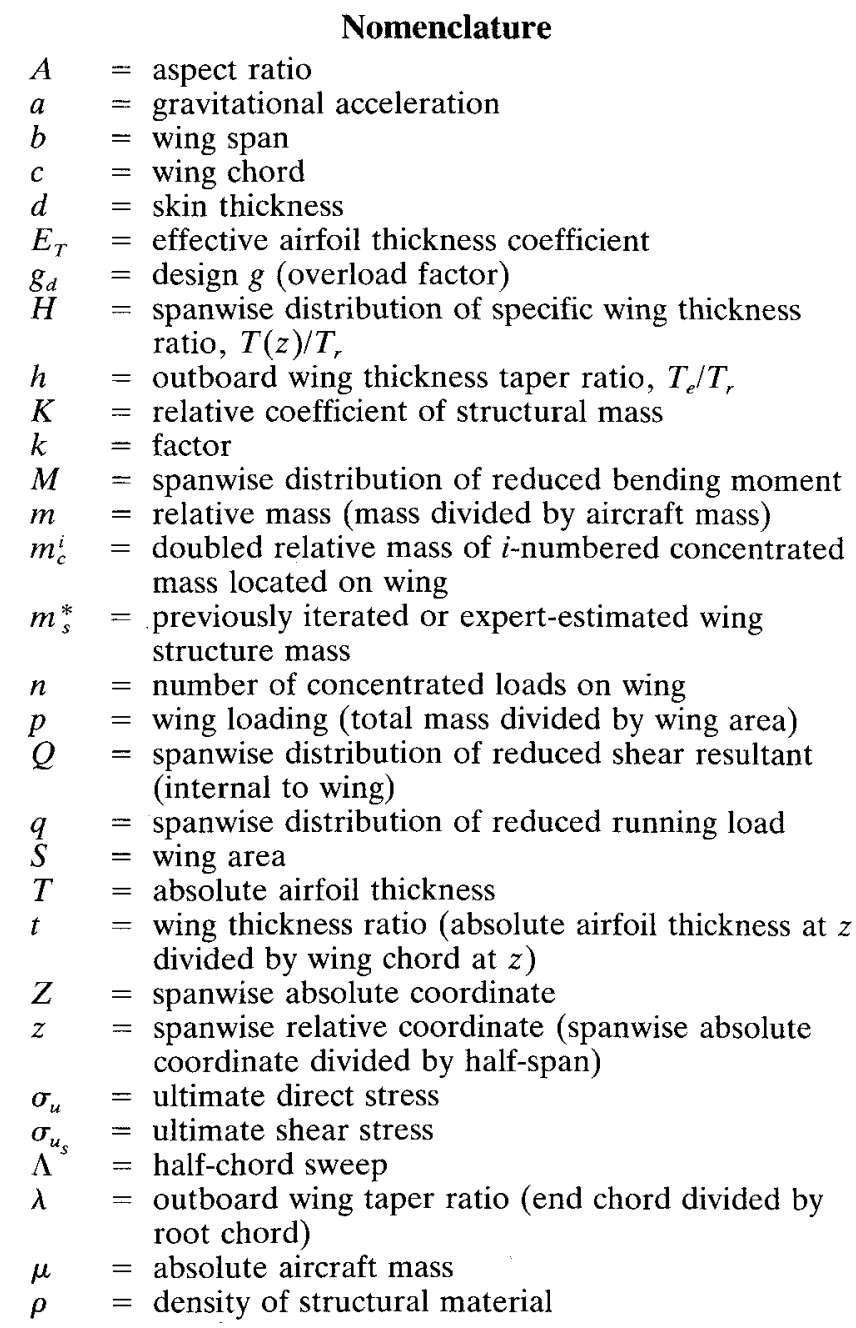

Received Dec. 13, 1990; revision received Oct. 15, 1991; accepted for publication Oct. 29, 1991. Copyright (c) 1991 by S. V. Udin and W. J. Anderson. Published by the American Institute of Aeronautics and Astronautics, Inc., with permission.

*Visiting Research Scholar, Aerospace Engineering Department; permanent affiliation: Graduate Student, Department 101, Moscow Aviation Institute, Volocolamsky shosse 4, Moscow 125080, Russia.

$\uparrow$ Professor of Aerospace Engineering, Aerospace Engineering Department. Senior Member AIAA. $\psi=$ outboard wing airfoil area taper ratio, $\approx c_{e} T_{e} / c_{r} T_{r}$ $=h \lambda=\lambda^{2}\left(t_{e} / t_{r}\right)$

Subscripts

$a=$ aerodynamic

abs $=$ absolute (not reduced) value

ail $=$ ailerons

$c=$ concentrated load

$e \quad=$ end of wing

$f \quad=$ fuselage

flap $=$ flaps

$\mathrm{fu}=$ fuel

$i \quad=$ inboard wing section

$M \quad=$ reduced bending moment

man $=$ manufacturing

mid $=$ middle

$o \quad=$ outboard wing section

$Q=$ reduced shear force

$r \quad=$ root of wing

rib $=$ ribs

$s \quad=$ wing structure

sk $=$ load-free wing skin

sl $=$ service life

tw $=$ twist moment

\section{Introduction}

$\mathbf{T}$ HERE are many advantages of twin fuselage aircraft (TFA) compared with conventional aircraft as range and payload increase. The theory of similarity predicts reduction of the payload capability when the dimensions of an aircraft are increased. ${ }^{1}$ Application of TFA can improve the situation. This scheme can be used for subsonic ${ }^{2,3}$ and supersonic aircraft. For the latter, the interference between fuselages improves drag characteristics. ${ }^{4} \mathrm{~A}$ multibody scheme has been considered for high-speed transport aircraft. ${ }^{5}$

The preliminary design of a TFA is not a simple task, because there is not much experience or a database for this aircraft type. Therefore, the mass formula for TFA must be derived with statistical coefficients that consider only common structural design characteristics such as the mass penalty for providing service life or the joint-mass penalty. ${ }^{1}$ The formula should consider all particulars of TFA in the computational model because there is no chance to improve the formula accuracy by comparing predicted results with experience. Another difficulty is the uncertainty of the flexible dynamic twist moment between fuselages. This moment depends on aircraft stiffness and capabilities of the asymmetric load aerodynamic compensation. The formula presented does not take into consideration flexible dynamic twist moment between fuselages. 
Usually designers do not trust a formula if they do not know which assumptions and simplifications were made during the formula derivation, therefore, a complete derivation is presented. There are no simplifications during the derivation, and so the resulting formula completely corresponds to the concept model. As a consequence, any differences between calculated mass and an actual wing mass may be clearly revealed. This will simplify formula improvement in the future. Additional details about the derivation of the wing mass formula are given in report form. 6,7

\section{Theoretical Method}

The relative mass of wing structure is a sum of components

$$
m_{s}=k_{\mathrm{sl}} k_{\mathrm{tw}} k_{\mathrm{man}}\left(m_{M}+m_{Q}\right)+m_{\mathrm{rib}}+m_{\mathrm{ail}}+m_{\mathrm{sk}}+m_{\mathrm{flap}}
$$

The manufacturing factor is defined ${ }^{1}$ as

$$
k_{\operatorname{man}}=1+k_{1}+k_{2}+k_{3}+k_{4}+k_{5}+k_{6}+k_{7}
$$

where $k_{i}$ have expert values within bounds presented in Table 1. This coefficient $k_{\text {man }}$ may be decreased as low as 1.3 for large, advanced-technology aircraft. ${ }^{1}$ The service life factor $k_{\mathrm{s} 1}$ is ultimate stress divided by panel fatigue stress.

The structural analysis is based on slender beam (EulerBernoulli) theory. The mass of elements required for twist moment is proportional to the mass required for bending moment. ${ }^{1}$ The twist moment mass has been considered by the twist moment factor $k_{\mathrm{tw}}$. It depends on the cosine of sweep, taper, and aspect ratio. For conventional aircraft, ${ }^{1}$ the formula for $k_{\mathrm{tw}}$ may be suggested

$$
k_{\mathrm{tw}}=1+\frac{0.015 \sqrt{A}(1+2 \lambda)}{(1+\lambda) \cos \Lambda}
$$

Usually the spanwise distribution of structure, fuel, and engine mass are considered through an "unloading coefficient." 1 Derivation of this coefficient will be more difficult if a complex form of wing or multibody fuselage scheme is used. The proposed approach implies consideration of the spanwise distribution of mass simultaneously with the lift distribution. The reduced quantities ${ }^{1}$ at any point $z$ are

$$
q=\frac{q_{\mathrm{abs}}(b / 2)}{a \mu G_{d}}, \quad Q=\frac{Q_{\mathrm{abs}}}{a \mu G_{d}}, \quad M=\frac{M_{\mathrm{abs}}}{a \mu G_{d}(b / 2)}
$$

Usually the approximate spanwise lift distribution $q_{a}$ has a standard form, exists in the preliminary design stage and may be considered at any spanwise point. Linear or quadratic approximation of the $q_{a}$ curve can be recommended. ${ }^{1}$ The reduced shear force $Q_{a}(z)$ and the reduced bending moment $M_{a}(z)$ due to aerodynamic load are then

$$
Q_{a}=\int_{z}^{1} q_{a} \mathrm{~d} z, \quad M_{a}=\int_{z}^{1} Q_{a} \mathrm{~d} z
$$

Table 1 Bounds on manufacturing coefficients

\begin{tabular}{lll}
\hline \hline & $\begin{array}{c}\text { Stepped thickness (rather than } \\
\text { tapered) }\end{array}$ & $0.10-0.13$ \\
$k_{1}$ & $\begin{array}{l}\text { Dead joint mass penalty } \\
k_{2}\end{array}$ & $0.15-0.30$ \\
$k_{3}$ & $\begin{array}{l}\text { Standard thickness of webs, ribs, } \\
\text { and other elements }\end{array}$ & $0.10-0.13$ \\
$k_{4}$ & $\begin{array}{l}\text { Joint fittings and joint defects } \\
k_{5}\end{array} \quad$ Plus tolerances & $0.10-0.15$ \\
$k_{6}$ & Manufacturing thicknesses & $0.04-0.09$ \\
$k_{7}$ & Breakdown joint mass penalty & $0.03-0.05$ \\
$k_{\text {man }}$ & Manufacturing factor & $0.10-0.20$ \\
\hline \hline
\end{tabular}

The fuel mass spanwise distribution $q_{f}$ depends on the airfoil area as taken by Badiagin. ${ }^{8}$ The wing structure mass spanwise distribution $q_{s}$ can be approximately obtained as a function of the chord spanwise distribution. The spanwise distributions of reduced shear and bending moment caused by a concentrated load (e.g., an engine) are

$$
\begin{array}{lll}
\text { for } 0<z<z_{c}: & Q_{c}=1, & M_{c}=z_{c}^{i}-z \\
\text { for } z_{c}<z<1: & Q_{c}=0, & M_{c}=0
\end{array}
$$

We define the relative coefficient of structural mass caused by shear and the relative coefficient of structural mass caused by bending moment

$$
\begin{aligned}
K_{Q} & =\int_{0}^{1}\left|\frac{Q_{\mathrm{sum}}}{\cos \Lambda}\right| \mathrm{d} z \\
& =\int_{0}^{1}\left|\frac{Q_{a}-Q_{\mathrm{fu}} m_{\mathrm{fu}}-Q_{s} m_{s}^{*}-\sum_{i=1}^{n} Q_{c}^{i} m_{c}^{i}}{\cos \Lambda}\right| \mathrm{d} z \\
K_{M} & =\int_{0}^{1}\left|\frac{M_{\mathrm{sum}}}{H \cos \Lambda}\right| \mathrm{d} z \\
& =\int_{0}^{1}\left|\frac{M_{a}-M_{\mathrm{fu}} m_{\mathrm{fu}}-M_{s} m_{s}^{*}-\sum_{i=1}^{n} M_{c}^{i} m_{c}^{i}}{H \cos \Lambda}\right| \mathrm{d} z
\end{aligned}
$$

The $K_{Q}$ and $K_{M}$ are the areas under the curves of relative shear and bending moment (Fig. 1). According to Eq. (4) the estimated relative mass of structure counteracting the shear and the estimated relative structural mass counteracting the bending moment (without consideration of manufacturing and service life) are

$$
\begin{aligned}
m_{Q} & =\frac{\rho}{\mu} \int_{0}^{b / 2} \frac{Q_{\mathrm{abs}}(Z)}{\sigma_{u_{s}} \cos \Lambda} \mathrm{d} Z=\frac{\rho g_{d} a}{\sigma_{u_{s}}} \frac{b}{2} \int_{0}^{1} \frac{Q_{\mathrm{sum}}}{\cos \Lambda} \mathrm{d} z \\
& =\frac{\rho g_{d} a}{2 \sigma_{u_{s}}} \sqrt{\frac{\mu A}{p}} K_{Q} \\
m_{M} & =2 \frac{\rho}{\mu} \int_{0}^{b / 2} \frac{E_{T} M_{\mathrm{abs}}(Z)}{T(Z) \sigma_{u} \cos \Lambda} \mathrm{d} Z \\
& =2 \frac{\rho g_{d} a}{\sigma_{u} T_{r}} \frac{b^{2}}{4} E_{T} \int_{0}^{1} \frac{M_{\mathrm{sum}}}{H \cos \Lambda} \mathrm{d} z \\
& =\frac{\rho g_{d} a}{2 \sigma_{u}} \frac{\mu}{p} A \frac{E_{T}}{T_{r}} K_{M}
\end{aligned}
$$

The coefficient $E_{T}$ considers the effective airfoil thickness and difference between loading on lower and upper wing panels. The approximate value of $E_{r}$ is $^{1}$

$$
E_{r} \approx 1.1\left(\frac{4 T_{2}}{T_{1}+2 T_{2}+T_{3}}\right)^{2} \approx 1.2-1.4
$$

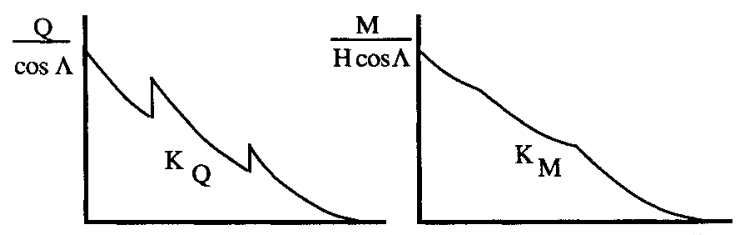

a) Relative spanwise coordinate, $z$ b) Relative spanwise coordinate, $z$

Fig. 1 Relative coefficients $K_{Q}$ and $K_{M}$. 
where $T_{1}$ is the first wing spar thickness, $T_{2}$ is the maximum airfoil thickness, and $T_{3}$ is the most rearward wing spar thickness. Actual operating stresses and twist moment are considered by factors in Eq. (1). If the upper and lower torsion box panels have been made from different materials then average values can be used

$$
\begin{aligned}
& \rho=\left[\left(\rho_{\text {upper panel }}+\rho_{\text {lower panel }}\right) / 2\right] \\
& \sigma=\left[\left(\sigma_{\text {upper panel }}+\sigma_{\text {lower panel }}\right) / 2\right]
\end{aligned}
$$

Masses of other elements (e.g., flaps and ailerons) may be taken from existing methods.

This theoretical derivation improves the accuracy of the wing mass formula by a more detailed calculation of mass spanwise distribution as compared to the method of Sheinin and Kozlowsky. ${ }^{1}$ It includes all physical relations that are important for optimization programs.

\section{Formula Derivation}

The geometry of a TFA wing is shown in Fig. 2. It is assumed that the inboard wing section is not tapered, and the inboard/outboard wing joint is located at the fuselage centerline.

\section{Spanwise Distribution of Reduced Aerodynamic Quantities}

The aerodynamic load spanwise distribution $q_{a}$ is related to the chord, ${ }^{1}$ and

$$
q_{a}(1)=\lambda q_{a}\left(z_{f}\right)
$$

According to Eq. (4) and using the equivalence of lift force to aircraft weight on the basis of TFA geometry, we obtain

$$
q_{a}\left(z_{f}\right) z_{f}+\frac{q_{a}\left(z_{f}\right)+q_{a}(1)}{2}\left(1-z_{f}\right)=1
$$

Using a linear equation, one takes the spanwise distribution of reduced running load

$$
\begin{aligned}
& \text { for } 0<z<z_{f} \text { (inboard wing): } \\
& q_{a}=\frac{2}{z_{f}(1-\lambda)+\lambda+1} \\
& \text { for } z_{f}<z<1 \text { (outboard wing): } \\
& q_{a}=2 \frac{\left[(1-z) /\left(1-z_{f}\right)\right](1-\lambda)+\lambda}{z_{f}(1-\lambda)+\lambda+1}
\end{aligned}
$$

The relative shear resultant $Q_{a}(z)$ due to aerodynamic force is

$$
\begin{aligned}
& \text { for } z_{f}<z<1: \quad Q_{a}=\int_{z}^{1} q_{a} \mathrm{~d} z \\
& =\frac{(1-\lambda)(1-z)^{2} /\left(1-z_{f}\right)+2 \lambda(1-z)}{z_{f}(1-\lambda)+\lambda+1}
\end{aligned}
$$

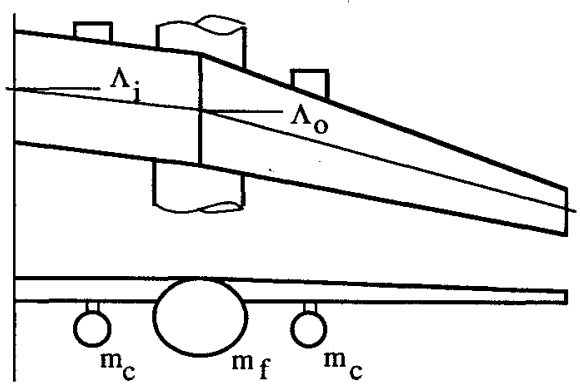

Fig. 2 TFA wing geometry.

$$
\begin{aligned}
& \text { for } \quad 0<z<z_{f}: \quad Q_{a}=\int_{z}^{z_{f}} q_{a} \mathrm{~d} z+Q_{a}\left(z_{f}\right) \\
& =1-\frac{2 z}{z_{f}(1-\lambda)+\lambda+1}
\end{aligned}
$$

The spanwise distribution of reduced bending moment due to aerodynamic force is

$$
\begin{aligned}
& \text { for } \quad z_{f}<z<1: \quad M_{a}=\int_{z}^{1} Q_{a} \mathrm{~d} z \\
& =\frac{\left[\frac{1}{3}(1-z)^{\left.3 /\left(1-z_{f}\right)\right](1-\lambda)+\lambda(1-z)^{2}}\right.}{z_{f}(1-\lambda)+\lambda+1} \\
& \text { for } \quad 0<z<z_{f}: \quad M_{a}=\int_{z}^{z_{f}} Q_{a} \mathrm{~d} z+M_{a}\left(z_{f}\right) \\
& =z_{f}-z-\frac{z_{f}^{2}-z^{2}-\frac{1}{3}\left(1-z_{f}\right)^{2}(1+2 \lambda)}{z_{f}(1-\lambda)+\lambda+1}
\end{aligned}
$$

The spanwise distributions of $q_{a}, Q_{a}$, and $M_{a}$ are shown in Fig. 3.

\section{Spanwise Distribution of Reduced Fuel Mass Quantities}

First, the formula for maximum fuel mass that may be located in the outboard wing, must be derived. The wing fuel volume is 8

$$
V=0.7 \frac{t_{\text {mid }} S_{o}^{1.5}}{\sqrt{A_{o}}} ; \quad t_{\text {mid }}=\frac{t_{r}+\lambda t_{e}}{1+\lambda}
$$

From geometric relations one may write

$$
S_{o}=S \frac{S_{o}}{S_{i}+S_{o}}=\frac{\mu}{p}\left[\frac{\left(1-z_{f}\right)(1+\lambda)}{2 z_{f}+\left(1-z_{f}\right)(1+\lambda)}\right]
$$

If the fuel is kerosene then the relative fuel mass in the outboard wing section is ${ }^{8}$

$$
m_{\mathrm{fu}}=800 \frac{V}{\mu}=560 \frac{t_{\mathrm{mid}}}{p^{1.5}} \sqrt{\frac{\mu}{A}}\left[\frac{\left(1-z_{f}\right)(1+\lambda)}{2 z_{f}+\left(1-z_{f}\right)(1+\lambda)}\right]^{1.5}
$$

This formula shows that for a heavy TFA it is possible to locate all fuel in the outboard wing, e.g., if $z_{f}\langle 0.35 ; \lambda\rangle$ $0.35 ; p<700 \mathrm{~kg} / \mathrm{m}^{2} ; m>400,000 \mathrm{~kg}$ then $m_{\mathrm{fu}}>0.3$ for all other useful parameters. Locating fuel in the fuselage is better than in the inboard wing because the fuel weight unloads the inboard wing section. We will assume that the inboard wing section does not contain fuel.

The spanwise distribution of reduced values caused by fuel weight must be derived. It is assumed that the fuel tank area $S_{\mathrm{fu}}(z)$ is proportional to the airfoil (wing cross section) area $S_{\text {air }}(z)$, and the airfoil area is proportional to the chord $c(z)$ multiplied by airfoil thickness $T(z)$. An approximation is made by assuming a linear relation (Fig. 4) for fuel distribution

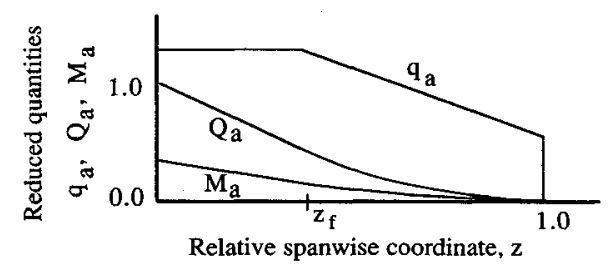

Fig. 3 Spanwise distribution of reduced quantities caused by aerodynamic force. 


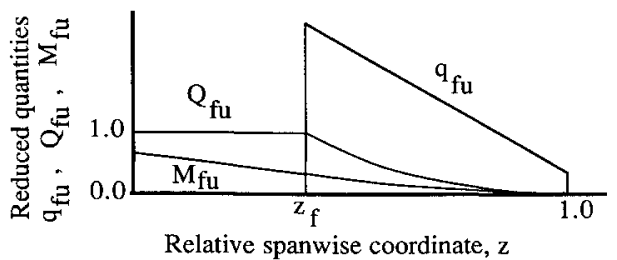

Fig. 4 Spanwise distribution of reduced quantities caused by fuel weight.

between the fuselage and wing tip. The extreme values are

$$
\frac{q_{\mathrm{fu}}\left(z_{f}\right)}{q_{\mathrm{fu}}(1)}=\frac{S_{\mathrm{fu}}\left(z_{f}\right)}{S_{\mathrm{fu}}(1)}=\frac{T_{r} c_{r}}{T_{e} c_{e}}=\frac{1}{\psi}
$$

where the area under the $q_{\mathrm{fu}}$ curve equals 1

$$
\frac{q_{\mathrm{fu}}\left(z_{f}\right)+q_{\mathrm{fu}}(1)}{2}\left(1-z_{f}\right)=1
$$

Then

$$
\begin{gathered}
q_{\mathrm{fu}}\left(z_{f}\right)=\frac{2}{(1+\psi)\left(1-z_{f}\right)} ; \quad q_{\mathrm{fu}}(1)=\frac{2 \psi}{(1+\psi)\left(1-z_{f}\right)} \\
\text { for } 0<z<z_{f}: \quad q_{\mathrm{fu}}=0
\end{gathered}
$$

$$
\text { for } z_{f}<z<1: q_{\mathrm{fu}}=2 \frac{(1-z)(1-\psi)+\left(1-z_{f}\right) \psi}{(\psi+1)\left(1-z_{f}\right)^{2}}
$$

Spanwise distribution of reduced shear force caused by fuel weight is

$$
\text { for } \begin{aligned}
& z_{f}<z<1: \quad Q_{\mathrm{fu}}(z)=\int_{z}^{1} q_{\mathrm{fu}} \mathrm{d} z \\
= & \frac{(1-z)^{2}(1-\psi)+2 \psi\left(1-z_{f}\right)(1-z)}{(\psi+1)\left(1-z_{f}\right)^{2}}
\end{aligned}
$$

$$
\text { for } 0<z<z_{f}: \quad Q_{\mathrm{fu}}(z)=\int_{z}^{z_{f}} q_{\mathrm{fu}} \mathrm{d} z+Q_{\mathrm{fu}}\left(z_{f}\right)=1
$$

Spanwise distribution of reduced bending moment caused by fuel weight is

$$
\begin{aligned}
& \text { for } z_{f}<z<1: \quad M_{\mathrm{fu}}(z)=\int_{z}^{1} Q_{\mathrm{fu}} \mathrm{d} z \\
& =\frac{\frac{1}{3}(1-z)^{3}(1-\psi)+\psi\left(1-z_{f}\right)(1-z)^{2}}{(\psi+1)\left(1-z_{f}\right)^{2}} \\
& \text { for } 0<z<z_{f}: \quad M_{\mathrm{fu}}(z)=\int_{z}^{z_{f}} Q_{\mathrm{fu}} \mathrm{d} z+M_{\mathrm{fu}}\left(z_{f}\right) \\
& =z_{f}-z+\frac{\left(1-z_{f}\right)(1+2 \psi)}{3 \psi+3}
\end{aligned}
$$

Figure 4 illustrates $q_{\mathrm{fu}}, Q_{\mathrm{fu}}$, and $M_{\mathrm{fu}}$.

Spanwise Distribution of Reduced Wing Structure Quantities

A linear approximation of wing mass spanwise distribution is recommended. ${ }^{1}$ For a conventional tapered wing, the reduced running load caused by structural weight $q_{s}^{*}$ is

$q_{s}^{*}(0)=(2+1.2 \lambda) /(\lambda+1) ; \quad q_{s}^{*}(1)=0.8 \lambda /(\lambda+1)$
A TFA has two different portions of wing with different tapers so that there are two subranges of wing mass spanwise distribution: inboard $q_{\mathrm{si}}(z)$ and outboard $q_{\mathrm{so}}(z)$. The proportions of TFA are taken as (parameters noted by asterisk relates to the conventional aircraft)

$$
\begin{aligned}
& \left(b^{*} / m_{s}^{*}\right) q_{s 1}^{*}(0)=\left(b_{i} / m_{\mathrm{si}}\right) q_{\mathrm{si}}\left(z_{f}\right) \\
& \left(b^{*} / m_{s}^{*}\right) q_{s 1}^{*}(1)=\left(b_{i} / m_{\mathrm{si}}\right) q_{\mathrm{si}}(0) \\
& \left(b^{*} / m_{s}^{*}\right) q_{s 2}^{*}(1)=\left(b_{o} / m_{\mathrm{so}}\right) q_{\mathrm{so}}(1) \\
& \left(b^{*} / m_{s}^{*}\right) q_{s 2}^{*}(0)=\left(b_{o} / m_{\mathrm{so}}\right) q_{\mathrm{so}}\left(z_{f}\right)
\end{aligned}
$$

Proceeding from the principle of smooth mass distribution, ${ }^{1}$ we write

$$
q_{\mathrm{si}}\left(z_{f}\right)=q_{\mathrm{so}}\left(z_{f}\right)
$$

The inboard wing taper ratio equals 1 , then

$$
\frac{m_{\mathrm{so}}}{m_{\mathrm{si}}}=\frac{b_{o} q_{s 1}^{*}(0)}{b_{i} q_{s 2}^{*}(0)}=\frac{\left(1-z_{f}\right)(1+\lambda)}{z_{f}(1.25+0.75 \lambda)}
$$

After some simplifications

$$
\begin{gathered}
q_{\mathrm{si}}\left(z_{f}\right)=q_{\mathrm{so}}\left(z_{f}\right)=\frac{8+4.8 \lambda}{z_{f}(1-\lambda)+4(1+\lambda)} \\
q_{\mathrm{so}}(1)=\frac{3.2 \lambda}{z_{f}(1-\lambda)+4(1+\lambda)} \\
q_{\mathrm{si}}(0)=\frac{2+1.2 \lambda}{z_{f}(1-\lambda)+4(1+\lambda)}
\end{gathered}
$$

A piecewise linear curve may be used to represent $q_{s}$

$$
\begin{aligned}
& \text { for } \quad z_{f}<z<1 \text { : } \\
& q_{s}=\frac{(8+1.6 \lambda)(1-z) /\left(1-z_{f}\right)+3.2 \lambda}{z_{f}(1-\lambda)+4(1+\lambda)}
\end{aligned}
$$

for $0<z<z_{f}$ :

$$
q_{s}=\frac{\left(z / z_{f}\right)(6+3.6 \lambda)+2+1.2 \lambda}{z_{f}(1-\lambda)+4(1+\lambda)}
$$

The reduced shear force caused by wing mass spanwise distribution is

$$
\begin{aligned}
& \text { for } z_{f}<z<1: \quad Q_{s}(z)=\int_{z}^{1} q_{s} \mathrm{~d} z \\
& =\frac{\left[(4+0.8 \lambda)(1-z)^{2} /\left(1-z_{f}\right)\right]+3.2 \lambda(1-z)}{z_{f}(1-\lambda)+4(1+\lambda)} \\
& \text { for } 0<z<z_{f}: \quad Q_{s}(z)=\int_{z}^{z_{f}} q_{s} \mathrm{~d} z+Q_{s}\left(z_{f}\right) \\
& =1-\frac{\left[3\left(z^{2} / z_{f}\right)+2 z\right](1+0.6 \lambda)}{z_{f}(1-\lambda)+4(1+\lambda)}
\end{aligned}
$$

The reduced bending moment caused by wing mass spanwise distribution is

$$
\begin{aligned}
& \text { for } z_{f}<z<1 \text { : } \\
& M_{s}=\frac{\left.\frac{1}{3}(4+0.8 \lambda)(1-z)^{3} /\left(1-z_{f}\right)\right]+1.6 \lambda(1-z)^{2}}{z_{f}(1-\lambda)+4(1+\lambda)}
\end{aligned}
$$




$$
\begin{aligned}
& \text { for } \quad 0<z<z_{f}: \\
& \qquad \begin{aligned}
M_{s} & =z_{f}-z \\
& +\frac{\left[\left(z^{2}-2 z_{f}^{2}+\left(z^{3} / z_{f}\right)\right](1+0.6 \lambda)+\left(\frac{4}{3}+1.867 \lambda\right)\left(1-z_{f}\right)^{2}\right.}{z_{f}(1-\lambda)+4(1+\lambda)}
\end{aligned}
\end{aligned}
$$

Figure 5 shows $q_{s}, Q_{s}$, and $M_{s}$.

\section{Spanwise Distribution of Reduced Concentrated Load Quantities}

The computational model has $n_{i}, i$-numbered inboard wing concentrated loads, and $n_{o}, j$-numbered outboard wing concentrated loads. The relative mass of such a load is $\frac{1}{2} m_{c}^{i}$ or $\frac{1}{2} m_{c}^{j}$ (i.e., $m_{c}^{i}$ or $m_{c}^{j}$ is the mass of both symmetric loads located in both halves of the wing). The relative coordinate of a concentrated load in the inboard wing section is $z_{c}^{i}$, and in the outboard wing $z_{c}^{j}$. Reduced shear and bending moment distributions caused by a concentrated load were presented in Eqs. (6) and (7).

The fuselage is also a concentrated load and for our case it is characterized by the relative mass

$$
m_{f}=1-m_{s}^{*}-m_{\mathrm{fu}}-\sum_{i=1}^{n_{i}} m_{c}^{i}-\sum_{j=1}^{n_{Q}} m_{c}^{j}
$$

\section{Spanwise Distribution of Specific Wing Thickness Ratio}

The inboard wing specific thickness ratio $H$ does not depend on $z$ and equals 1 . Using the linear equation for the outboard wing $\left(z_{f}<z<1\right)$

$$
H=\frac{1}{T_{r}}\left(\frac{1-z}{1-z_{f}}\left(T_{r}-T_{e}\right)+T_{e}\right)=\frac{1-z}{1-z_{f}}(1-h)+h
$$

$T_{r}$ must be derived. The wing area is

$$
\begin{aligned}
S & =S_{o}+S_{i}=\frac{c_{r}+c_{e}}{2} b\left(1-z_{f}\right)+c_{r} b z_{f} \\
& =c_{r} b\left(\frac{1+\lambda}{2}\left(1-z_{f}\right)+z_{f}\right)
\end{aligned}
$$

where

$$
S=\mu / p ; \quad b=\sqrt{S A}
$$

and then

$$
T_{r}=t_{r} c_{r}=\frac{2 t_{r}}{(1+\lambda)\left(1-z_{f}\right)+2 z_{f}} \sqrt{\frac{\mu}{p A}}
$$

\section{Structural Mass Required by Shear Force}

The integral in Eq. (8) must be represented for the inboard

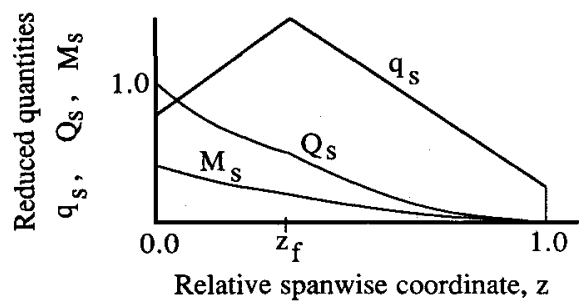

Fig. 5 Spanwise distribution of reduced quantities caused by structural weight. and outboard wing as

$$
K_{Q}=K_{Q_{i}}+K_{Q_{o}}
$$

where

$$
\begin{array}{r}
K_{Q_{i}}=\frac{1}{\cos \Lambda_{i}} \int_{0}^{z_{f}} \mid Q_{a}-Q_{\mathrm{fu}} m_{\mathrm{fu}}-Q_{s} m_{s}^{*} \\
-Q_{f} m_{f}-\sum_{j=1}^{n_{O}} Q_{c}^{j} m_{c}^{j}-\sum_{i=1}^{n_{i}} Q_{c}^{i} m_{c}^{i} \mid \mathrm{d} z
\end{array}
$$

Usually the integrand in Eq. (53) is negative in the range (0, $z_{f}$ ) for TFA (Fig. 6). For an analytical integration, one desires that the quantity within the absolute brackets does not change sign in the range of integration so that the integral of absolute value is the absolute value of the integral. This assumption will be discussed later. Using Eqs. (8), (19), (31), and (44), we have

$$
\begin{aligned}
K_{Q_{i}} & =\frac{1}{\cos \Lambda_{i}}\left[\frac{z_{f}^{2}}{z_{f}(1-\lambda)+\lambda+1}\right. \\
& \left.-\frac{z_{f}^{2}(2+1.2 \lambda) m_{s}^{*}}{z_{f}(1-\lambda)+4 \lambda+4}+\sum_{i=1}^{n_{i}}\left(z_{f}-z_{c}^{i}\right) m_{c}^{i}\right]
\end{aligned}
$$

In the same manner

$$
K_{Q_{o}}=\frac{1}{\cos \Lambda_{o}} \int_{z_{f}}^{1}\left|Q_{a}-Q_{\mathrm{fu}} m_{\mathrm{fu}}-Q_{s} m_{s}^{*}-\sum_{j=1}^{n_{o}} Q_{c}^{j} m_{\mathrm{c}}^{j}\right| \mathrm{d} z
$$

Assume that the quantities within the absolute brackets are positive (Fig. 6). Further, according to Eqs. (7), (18), (30), (43), and (47), we obtain

$$
\begin{aligned}
K_{Q_{o}} & =\frac{1}{\cos \Lambda_{o}}\left[\frac { ( 1 - z _ { f } ) ^ { 2 } } { 3 } \left(\frac{1+2 \lambda}{z_{f}(1-\lambda)+\lambda+1}\right.\right. \\
& \left.-\frac{(1+2 \psi) m_{\mathrm{fu}}}{(\psi+1)\left(1-z_{f}\right)}-\frac{(4-5.6 \lambda) m_{s}^{*}}{z_{f}(1-\lambda)+4 \lambda+4}\right) \\
& \left.-\sum_{j=1}^{n_{j}}\left(z_{c}^{j}-z_{f}\right) m_{c}^{j}\right]
\end{aligned}
$$

According to Eq. (10), estimated structural mass required by shear (without consideration of manufacturing and service life) is

$$
m_{Q}=\frac{\rho g_{d} a}{2 \sigma_{u_{s}}} \sqrt{\frac{\mu A}{p}}\left(K_{Q_{i}}+K_{Q_{o}}\right)
$$

The actual operating stresses are considered in Eq. (1).

\section{Structural Mass Required by Bending Moment}

The integral in Eq. (9) can be represented as

$$
K_{M}=K_{M_{i}}+K_{M_{o}}
$$

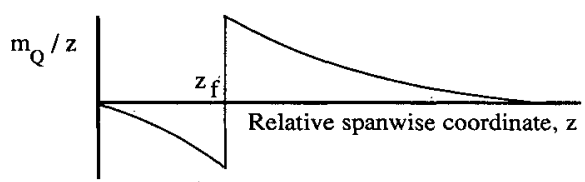

Fig. 6 Mass distribution required by shear force. 
where

$$
K_{M_{i}}=\int_{0}^{z_{f}}\left|\frac{M_{a}-M_{\mathrm{fu}} m_{\mathrm{fu}}-M_{s} m_{s}^{*}-M_{f} m_{f}-\sum_{j=1}^{n_{Q}} M_{c}^{j} m_{c}^{j}-\sum_{i=1}^{n_{i}} M_{c}^{i} m_{c}^{i}}{H \cos \Lambda_{i}}\right| \mathrm{d} z
$$

The quantity within the absolute brackets is assumed positive. Using Eqs. (9), (21), (33), (46), and (47) one has

$$
\begin{aligned}
K_{M_{i}} & =\frac{z_{f}}{\cos \Lambda_{i}}\left[\frac{\left(1-z_{f}\right)^{2}(1+2 \lambda)-2 z_{f}^{2}}{3\left(z_{f}(1-\lambda)+\lambda+1\right)}\right. \\
& -\frac{\left(1-z_{f}\right)(1+2 \psi) m_{\mathrm{fu}}}{3 \psi+3} \\
& -\frac{\left(\frac{4}{3}+1.87 \lambda\right)\left(1-z_{f}\right)^{2}-\frac{17}{12} z_{f}^{2}(1+0.6 \lambda)}{z_{f}(1-\lambda)+4(\lambda+1)} m_{s}^{*} \\
& \left.-\sum_{j=1}^{n_{o}} z_{c}^{j} m_{c}^{j}-\sum_{i=1}^{n_{i}} \frac{\left(z_{c}^{i}\right)^{2}-z_{f}^{2}}{2 z_{f}} m_{c}^{i}\right]
\end{aligned}
$$

In the same manner

$$
K_{M_{o}}=\int_{z_{f}}^{1}\left|\frac{M_{a}-M_{\mathrm{fu}} m_{\mathrm{fu}}-M_{s} m_{s}^{*}-\sum_{j=1}^{n_{o}} M_{c}^{j} m_{c}^{j}}{H \cos \Lambda_{o}}\right| \mathrm{d} z
$$

Using Eqs. (8), (20), (32), and (45), one may write

$$
\begin{aligned}
K_{M_{o}} & =\frac{1-z_{f}}{(1-h) \cos \Lambda_{o}}\left\{\frac { ( 1 - z _ { f } ) ^ { 2 } } { 3 ( 1 - h ) ^ { 3 } } \left(\frac{1}{3}-\frac{3}{2} h\right.\right. \\
& \left.+3 h^{2}-\frac{11}{6} h^{3}+h^{3} \log h\right)\left(\frac{1-\lambda}{z_{f}(1-\lambda)+\lambda+1}\right. \\
& \left.-\frac{(1-\psi) m_{\mathrm{fu}}}{(1+\psi)\left(1-z_{f}\right)}-\frac{(4+0.8 \lambda) m_{s}^{*}}{z_{f}(1-\lambda)+4+4 \lambda}\right) \\
& +\left(\frac{\left.1-z_{f}\right)^{2}\left(\frac{1}{2}-2 h+\frac{3}{2} h^{2}-h^{2} \log h\right)}{}\right. \\
& \cdot\left(\frac{\lambda}{z_{f}(1-\lambda)+\lambda+1}-\frac{\psi m_{\mathrm{fu}}}{(\psi+1)\left(1-z_{f}\right)}\right. \\
& \left.-\frac{1.6 \lambda m_{s}^{*}}{z_{f}(1-\lambda)+4 \lambda+4}\right)-\sum_{j=1}^{n_{o}} m_{c}^{i}\left[z_{c}^{i}-z_{f}\right. \\
& -\left(h \frac{1-z_{f}}{h-1}-1+z_{c}^{j}\right) \log \left(\frac{1-z_{c}^{j}}{1-z_{f}}\right. \\
& \cdot(1-h)+h)]\}
\end{aligned}
$$

a) Concept model corresponds to actual mass distribution

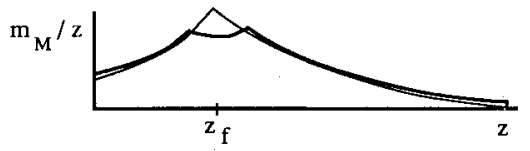

b) Concept model does not correspond to actual mass distribution

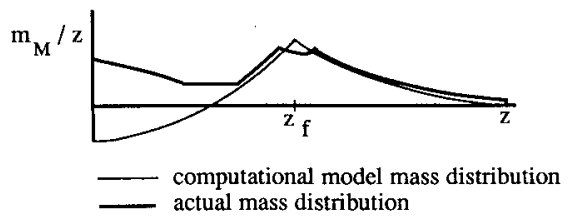

Fig. 7 Fuselage location effect upon mass needed to carry bending moment.
Assume that $h \neq 1$. Otherwise division by zero will occur. If the formula is to be used for a nontapered wing, the integral in Eq. (61) must be rederived with $H=1$.

Using Eqs. (11) and (51) one can estimate mass of elements caused by bending moment without consideration of manufacturing and service life:

$m_{M}=\frac{\rho g_{d} a}{2 \sigma_{u}} A^{1.5} \sqrt{\frac{\mu}{p}} E_{T} \frac{(\lambda+1)\left(1-z_{f}\right)}{2 t_{r}}\left(K_{M_{i}}+K_{M_{o}}\right)$

The actual operating stresses are considered in Eq. (1).

\section{Twist Moment Factor for TFA}

The TFA wing has two sections with different sweep and taper. On the basis of Eq. (3) we have

$$
k_{t w}=1+\frac{0.0225 \sqrt{b^{2} / S_{i}}}{\cos \Lambda_{i}}+\frac{0.015 \sqrt{b_{o}^{2} / S_{o}}(1+2 \lambda)}{(\lambda+1) \cos \Lambda_{o}}
$$

After simplification

$$
\begin{aligned}
k_{t w} & =1+\sqrt{A\left[2 z_{f}+\left(1-z_{f}\right)(1+\lambda)\right]} \\
\cdot & {\left[\frac{0.0225}{\cos \Lambda_{i}} \sqrt{\frac{z_{f}}{2}}+\frac{0.015(1+2 \lambda) \sqrt{1-z_{f}}}{(\lambda+1)^{1.5} \cos \Lambda_{o}}\right] }
\end{aligned}
$$

\section{Range of Formula Application}

It has been assumed that quantities within absolute brackets in Eqs. (53), (55), (59), and (61) are positive. But in some cases, this is not true. The spanwise distribution of mass needed to carry bending moment is shown in Fig. 7 in accordance with both the concept model and the actual physical bending moment mass. There is a difference between actual and formula mass when $z_{f}$ increases. The bounds of formula applicability in terms of $z_{f}$ must be obtained.

The wing root skin thickness is assumed to be set by strength requirements and is greater than minimum thickness requirements for manufacturing, otherwise, the formula must not be used. We require

$$
d_{t}<\frac{a \mu G_{d} E_{T} b M_{r}}{c_{t b} k_{\mathrm{rib}} T_{r} \sigma_{u} k_{\operatorname{man}} k_{\mathrm{sl}} k_{\mathrm{tw}}}
$$

where $k_{\text {rib }}$ defined by Sheinin and Kozlowsky ${ }^{1}$ (approximate value is 2 ); $c_{\mathrm{tb}}$-torsion box chord (approximate value is $\frac{1}{2} c_{r}$, $c_{r}$ in Eq. (51)). According to Eqs. (4), (6), (7), (22), (32), (33), and (45-47), one may write

$$
\begin{aligned}
d_{t} & <\frac{a g_{d} \sqrt{\mu p A^{3}} E_{T}\left[(\lambda+1)\left(1-z_{f}\right)+2 z_{f}\right]^{2}}{4 t_{r} \sigma_{u} k_{\mathrm{man}} k_{\mathrm{si}} k_{\mathrm{tw}}} \\
& \cdot\left[\frac{\frac{1}{3}\left(1-z_{f}\right)^{2}(1+2 \lambda)-z_{f}^{2}}{z_{f}(1-\lambda)+1+\lambda}-\frac{\left(1-z_{f}\right)(1+2 \psi)}{3 \psi+3} m_{\mathrm{fu}}\right. \\
& -\frac{\left(\frac{4}{3}+1.886 \lambda\right)\left(1-z_{f}\right)^{2}-2 z_{f}^{2}(1+0.6 \lambda)}{z_{f}(1-\lambda)+4 \lambda+4} m_{s}^{*} \\
& \left.-\sum_{i=1}^{n_{i}} m_{c}^{i}\left(z_{c}^{i}-z_{f}\right)-\sum_{j=1}^{n_{o}} m_{c}^{i}\left(z_{c}^{j}-z_{f}\right)\right]
\end{aligned}
$$


a) Concept model corresponds to actual mass distribution

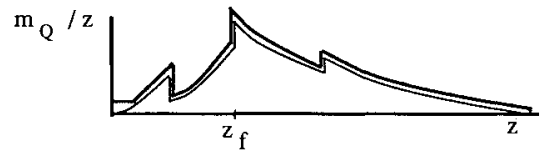

b) Concept model does not correspond to actual mass distribution

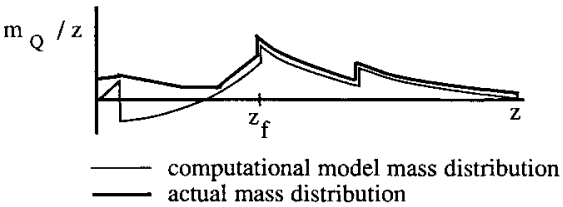

Fig. 8 Fuselage location effect upon mass needed to carry shear resultant.

Equation (67) involves the 4th power of $z_{f}$. Results may be obtained by a numerical method. A computer program to solve Eq. (67) indicates that for $z_{f} \leq 0.3, \lambda \geq 0.35, m_{0} \geq$ $100,000 \mathrm{~kg}, d_{t} \leq 1.5 \mathrm{~mm}$, and $A \geq 10$ the inequality is true for all other useful parameters. If the actual parameters of a multibody aircraft are outside these limits, then verification of use of the formula must be carried out. The inequality is always true for a single-body aircraft

The spanwise distributions of relative mass needed to carry shear, in accordance with both the concept model and the actual bending moment mass, are shown on Fig. 8. There is a difference between actual and calculated mass if concentrated forces are located near the longitudinal aircraft axis. The simple relation ${ }^{1}$ between the mass caused by bending moment, and the mass caused by shear force for such cases is

$$
K_{Q}=0.1 K_{M}
$$

This formula also can be recommended for moderate-sized aircraft. The mass caused by shear is approximately $\frac{1}{20}$ of the total wing mass and its influence on the formula accuracy is not significant. If the outboard wing is not tapered, then Eq. (61) must be rederived.

\section{Mass of Other Wing Elements}

The mass of other elements may be found by existing methods. Some formulae are presented below.

\section{Mass of Ribs}

The mass of ribs for TFA is

$$
m_{\mathrm{rib}}=m_{\text {rib }}+2 m_{\text {frib }}+m_{\text {row rib }} \approx 0.15 m_{M}
$$

where $m_{r \text { rib }}$ is root rib mass; $m_{f \text { rib }}$ is fuselage rib mass; and $m_{\text {row rib }}$ is row rib mass (that are only aerodynamically loaded). The relative mass of conventional aircraft root ribs $m_{r \text { rib }}^{*}$ is ${ }^{1}$

$$
m_{r \text { rib }}^{*}=\left(\frac{1.05 \times 10^{-4}}{\mu}\right)\left(\frac{M_{r}}{a}\right) \frac{c_{r}}{2} \sin \Lambda
$$

where $M_{r}$ is the root bending moment $(\mathrm{Nm})$. For twin fuselage aircraft bending moments $(N m)$ at $z=0$ and $z=z_{f}$ are

$$
\begin{aligned}
M_{r}(0) & =a \frac{b}{2} \mu g_{d} M(0)=a \frac{b}{2} \mu g_{d} \int_{0}^{1} Q \mathrm{~d} z \\
M_{r}\left(z_{f}\right) & =a \frac{b}{2} \mu g_{d} M\left(z_{f}\right)=a \frac{b}{2} \mu g_{d} \int_{z_{f}}^{1} Q \mathrm{~d} z
\end{aligned}
$$

These integrals were seen previously in Eqs. (53) and (54). Considering the sense of the moment, and using Eqs. (51) and (71), we obtain

$$
\begin{aligned}
m_{r \text { rib }} & =\frac{1.05 \times 10^{-4} g_{d}\left|K Q_{o} \cos \Lambda_{o}-K Q_{i} \cos \Lambda_{i}\right|}{(1+\lambda)\left(1-z_{f}\right)+2 z_{f}} \\
\cdot \frac{\mu}{p} \sqrt{\frac{A}{a}} \sin \left|\Lambda_{i}\right| & \cdot \\
m_{f \text { rib }} & =\frac{1.05 \times 10^{-4} g_{d} K Q_{o} \cos \Lambda_{o}}{(1+\lambda)\left(1-z_{f}\right)+2 z_{f}} \frac{\mu}{p} \sqrt{\frac{A}{a}} \sin \left|\Lambda_{o}-\Lambda_{i}\right|
\end{aligned}
$$

Note that these formulae do not correspond to the theory of similarity that implies the relative mass varies as mass $\mu$ to the $\frac{1}{2}$ power. The initial formula Eq. (70) also does not correspond to the theory of similarity.

The row rib mass is ${ }^{1}$

$$
m_{\text {row rib }}=\left[0.26 \sqrt{S_{t b}}+0.0006\left(g_{d} \mu / S\right)\right] S_{t b}
$$

where $S_{t b}$ is the torsion box area. Using some simple relations we have

$$
m_{\text {row rib }}=0.0919 \sqrt{\mu / p^{3}}+0.0003 g_{d}
$$

Mass of Forward and Rear Parts of Wing Skin

The mass of forward and rear parts of wing skin (not loaded surfaces) in accordance with experience ${ }^{\mathbf{1}}$ is

$$
m_{\mathrm{sk}}=(3 / p)
$$

Mass of Flaps

The mass of flaps consists of components

$$
m_{\text {flap }}=m_{\text {lef }}+m_{\text {tef }}+m_{\text {of }}
$$

The relative mass of leading edge flaps is ${ }^{1}$

$$
m_{\text {lef }}=\frac{k_{\text {lef }}}{p}
$$

where $K_{\text {lef }}$ for a Krueger flap is 2.5 , and for others is 3.5 . The relative mass of trailing-edge flaps, in accordance with the Torenbeek formula ${ }^{1}$ is

$$
m_{\mathrm{tef}}=\frac{2.706}{\mu} S_{f} k_{f}\left(S_{f} b_{f}\right)^{3 / 16}\left[\left(\frac{V_{f}}{100}\right)^{2} \frac{\sin a_{f} \cos \Lambda_{f}}{t_{f}}\right]^{3 / 4}
$$

where $S_{f}$ is trailing edge flaps area, $k_{f}$ is the statistical coefficient, $b_{f}$ is the flap length (equals spanwise flap length divided by the cosine of the trailing edge sweep), $V_{f}$ is flapdown speed, $a_{f}$ is flap deflection angle, $\Lambda_{f}$ is half chord flap sweep, $t_{f}$ is thickness ratio of upper flap, $k_{f}$ for double-slotted flap is 1.05 , for triple-slotted flap is 1.25 , for triple-slotted extending split flap is 1.6, for single-slotted Fowler flap is 1.25 , for double-slotted Fowler flap is 1.3 , and for tripleslotted Fowler flap is 1.62. For the relative mass of other flap types (brake flaps, spoilers) it is suggested that

$$
m_{\mathrm{of}}=\left(30 \tilde{S}_{\mathrm{of}}\right) / p
$$

where $\tilde{S}_{\text {of }}$ is the relative area of flap (area of flap divided by area of wing).

\section{Mass of Ailerons}

The relative mass of ailerons is ${ }^{1}$

$$
m_{\text {ail }}=0.03 \bar{S}_{\text {ail }} ; \quad \tilde{S}_{\text {ail }}=\frac{S_{\text {ail }}}{S}
$$

One can also use Eq. (80) for ailerons. 
Table 2 Results of formula usage for conventional aircraft

\begin{tabular}{lrcccrr}
\hline \hline Aircraft & $\mu, \mathrm{kg}$ & $m_{s}$, actual & $k_{\operatorname{man}}$ & $m_{s}$, formula & Error, \% & Error, ${ }^{10} \%$ \\
\hline B-727-100 & 72,600 & 0.111 & 1.9 & 0.1206 & 8.7 & -0.7 \\
B-747-100 & 322,000 & 0.122 & 1.7 & 0.1061 & -13.1 & -13.3 \\
DC-9-30 & 49,000 & 0.106 & 1.9 & 0.1018 & -4.0 & -6.5 \\
DC-10-10 & 195,000 & 0.114 & 1.8 & 0.1069 & -6.2 & 10.4 \\
DC-10-30 & 252,000 & 0.106 & 1.8 & 0.0989 & -6.7 & 2.4 \\
A-300-B2 & 137,700 & 0.145 & 1.7 & 0.1423 & -1.8 & -14.6 \\
C-5A & 349,000 & 0.130 & 1.7 & 0.1349 & 3.8 & -1.3 \\
Tu-154 & 90,000 & 0.102 & 1.9 & 0.1085 & 6.4 & 19.5 \\
An-10 & 54,000 & 0.0981 & 1.9 & 0.0929 & -5.3 & 0.3 \\
An-22 & 250,000 & 0.119 & 1.8 & 0.1167 & -1.9 & -9.1 \\
An-24 & 21,000 & 0.1142 & 1.9 & 0.1189 & 4.1 & 16.0 \\
Il-76-T & 171,000 & 0.121 & 1.8 & 0.1351 & 11.7 & -14.9 \\
\hline \hline
\end{tabular}

\section{Applications}

The wing structural mass for TFA is obtained from Eq. (1) where mass components are defined in Eqs. (57), (63), (69), (76-78), (80), and (81). The twist moment factor for the TFA is Eq. (65). The formula should not be used if initial parameters do not correspond to Eq. (67). If the wing has a concentrated load near the longitudinal aircraft axis, Eq. (54) must be replaced by Eq. (68).

\section{Conventional Single-Body Aircraft}

The mass formula [Eq. (1)] can be used for conventional aircraft if $z_{f}$ equals the fuselage radius. The fuselage (inboard) wing-section geometry and loading are completely considered in this case. The twist moment factor is Eq. (3).

Results for several single body aircraft ${ }^{9}$ are presented in Table 2 . The accuracy is within $(-13.1,+11.7 \%)$ and $\mathrm{rms}$ error is $7.0 \%$. The manufacturing factors were chosen through engineering judgement without access to manufacturing data. The chosen factors were not altered to drive the error to zero. (Otherwise, all errors could be made zero!)

For comparison, single-body results using the Torenbeek formula for class II estimation ${ }^{10}$ are shown as the last column in Table 2. The accuracy is within $(-14.9,+19.5 \%)$ and rms error is $11.1 \%$. This equation is not as accurate as the equation developed above, but has the advantage of being simpler.

Details of the examples, comparisons with the Badiagin formula, ${ }^{1}$ and spreadsheet calculations, are included in Ref. 7. These will be of help to the practicing engineer.

\section{Other Unconventional Schemes}

The formula can also be used for aircraft with three or more bodies, if Eq. (67) is true. The fuselage loads are represented by concentrated loads in this case. Use of Eq. (68) instead of Eq. (53) is recommended. The Voyager ${ }^{9}$ corresponds to this case. The formula can also be used to calculate mass required to carry bending moment for the multibody scheme for supersonic aircraft. ${ }^{5}$

\section{References}

${ }^{1}$ Sheinin, V. M., and Kozlowsky, V. I., Weight Design And Effectivity of Passenger Aircraft, (in Russian), 2nd ed., Mashinostroenie, Moscow, 1984.

${ }^{2}$ Roskam, J., Airplane Design: Part 2, Preliminary Configuration Design and Integration of the Propulsion System, Roskam Aviation and Engineering Corp., Ottawa, KS, 1985.

${ }^{3}$ Houbolt, J. C., "Why Twin-Fuselage Aircraft?" Journal of Astronautics and Aeronautics, Vol. 20, No. 4, 1982, p. 26.

${ }^{4}$ Wood, R. M., Miller, D. S., and Brentner, K. S., "Theoretical and Experimental Investigation of Supersonic Aerodynamic Characteristics of a Twin-Fuselage Concept," NASA TP-2184, Aug., 1983.

${ }^{5}$ MacKinnon, M. I. K., Ball, D. N., Hallstaff et al., "High-Speed Civil Transport Study," NASA CR 4233, 4234, 1989.

"Udin, S. V., and Anderson, W. J., "A Method of Wing Mass Formula Derivation," Univ. of Michigan, Aerospace Engineering Rept. SM-90.1, Ann Arbor, MI, Nov. 1990.

'Udin, S. V., Anderson, W. J., "The Complete Derivation of Wing Mass Formula for Twin Fuselage Aircraft," Univ. of Michigan, Aerospace Engineering Rept. SM-90.2, Ann Arbor, MI, Nov. 1990.

${ }^{8}$ Badiagin, A. A., Eger, S. M., and Mishin, W. F., Aircraft Design, (in Russian) Mashinostroenie, Moscow, 1972.

${ }^{9}$ Taylor, J. W. R., Jane's All The World Aircraft 1986-1987, Jane's Publ. Co., London, 1987.

${ }^{10}$ Roskam, J., Airplane Design: Part V, Component Weight Estimation, Roskam Aviation and Engineering Corp., Ottawa, KS, 1985, p. 69 . 